\title{
Complex Shepard Operators and Their Summability
}

\author{
Oktay Duman॰ and Biancamaria Della Vecchia@
}

\begin{abstract}
In this paper, we construct the complex Shepard operators to approximate continuous and complex-valued functions on the unit square. We also examine the effects of regular summability methods on the approximation by these operators. Some applications verifying our results are provided. To illustrate the approximation theorems graphically we consider the real or imaginary part of the complex function being approximated and also use the contour lines of the modulus of the function.
\end{abstract}

Mathematics Subject Classification. 30E10, 40A25, $40 \mathrm{C} 05$.

Keywords. Approximation in the complex plain, Shepard operators, matrix summabiliy methods, Cesàro summability.

\section{Introduction}

In 1968, Donald Shepard introduced a sequence of operators that was highly effective in scattered data interpolation problems, which are known in the literature as Shepard operators [29]. Since these operators are of interpolatory-type, linear and positive, they are also quite useful in the classical approximation theory. Recall that the Shepard interpolatory operators are defined by

$$
S_{n, \lambda}(f ; x)=\frac{\sum_{k=0}^{n}\left|x-\frac{k}{n}\right|^{-\lambda} f\left(\frac{k}{n}\right)}{\sum_{k=0}^{n}\left|x-\frac{k}{n}\right|^{-\lambda}}, \quad \lambda \geq 1, \mathrm{n} \in \mathbb{N}, x \in[0,1]
$$

for an arbitrary continuous real-valued function $f$ on the unit interval $[0,1]$. These operators defined on real-valued functions have been studied extensively. For example, error estimates, direct and converse theorems, saturation results, rational approximation, simultaneous approximation may be found in 


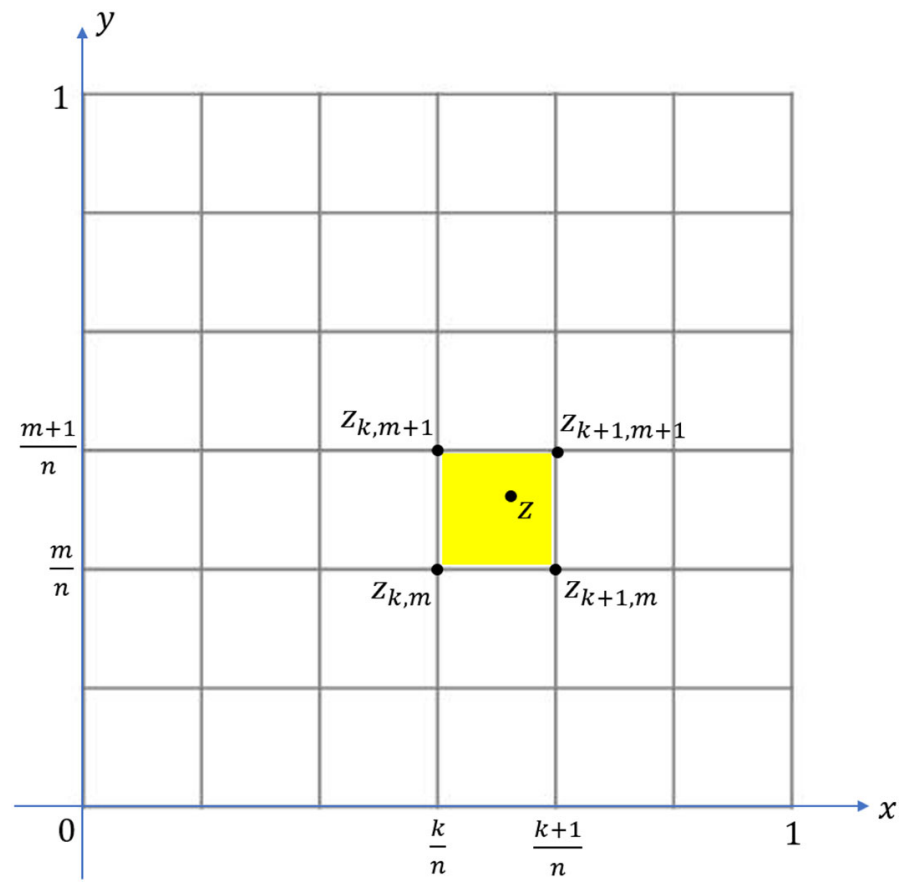

Figure 1. Sample points $z_{k, m}$

[14-16,31,34-36]. There are also several modifications of the original Shepard operators in order to increase the accuracy of approximation or to solve specific interpolation problems in CAGD such as scattered data and image compression $[2,6,7,9,10,17-22,24]$. However, to the best of our knowledge, there is a limited information in the literature about a complex version of Shepard operators. By using the fundamental polynomials of Lagrange interpolation at the roots of unity, Hermann studied Shepard-type operators for functions on the unit circle [27].

In the present paper we mainly introduce the complex Shepard operators and systematically investigate their approximation properties. We also examine the effects of regular summability methods on the approximation by these operators. To visualize the corresponding approximation we consider the real or imaginary part of the complex function being approximated and also use the contour lines of the modulus of the complex function. 


\section{Approximation by the Complex Shepard Operators}

Let $K$ denote the unit square, i.e.,

$$
K=[0,1] \times[0,1]=\{z=x+i y \in \mathbb{C}: x, y \in[0,1]\},
$$

where $i^{2}=-1, x=\Re(z)$ and $y=\Im(z)$. Now for a given $n \in \mathbb{N}$, consider the following sample points for the set $K$ :

$$
z_{k, m, n}:=z_{k, m}=\frac{k}{n}+i \frac{m}{n}, \mathrm{k}, \mathrm{m} \in\{0,1, \ldots, n\}
$$

(see Fig. 1).

Let $f$ be a complex-valued function defined on $K$. Then, for a positive real number $\lambda$, we define the complex Shepard operators by

$$
\mathbb{S}_{n, \lambda}(f ; z)=\frac{\sum_{k, m=0}^{n}\left|z-z_{k, m}\right|^{-\lambda} f\left(z_{k, m}\right)}{\sum_{k, m=0}^{n}\left|z-z_{k, m}\right|^{-\lambda}},
$$

where we write $\sum_{k, m=0}^{n}$ for the double summation $\sum_{k=0}^{n} \sum_{m=0}^{n}$. Observe that $\mathbb{S}_{n, \lambda}(f)$ interpolates at the sample points $z_{k, m}$, i.e.,

$$
\mathbb{S}_{n, \lambda}\left(f ; z_{k, m}\right)=f\left(z_{k, m}\right) \text { for } k, m \in\{0,1, \ldots, n\} .
$$

We denote the space of all continuous and complex-valued functions on $K$ by $C(K, \mathbb{C})$. Then, for the complex Shepard operators defined by (1) we get the following approximation result.

Theorem 1. For every $f \in C(K, \mathbb{C})$ and $\lambda \geq 3$, we have

$$
\mathbb{S}_{n, \lambda}(f) \rightrightarrows f \text { on } K,
$$

where, as usual, the symbol $\rightrightarrows$ denotes the uniform convergence.

To prove Theorem 1, we first need the following lemma.

Lemma 1. Let $z \in K$ with $z \neq z_{k, m}$. Then, for every $n \in \mathbb{N}$ and $\lambda>0$,

$$
\left(\sum_{k, m=0}^{n}\left|z-z_{k, m}\right|^{-\lambda}\right)^{-1}=O\left(n^{-\lambda}\right)
$$

holds.

Proof. Define the indices $k^{*}$ and $m^{*}$ by

$$
\left|z-z_{k^{*}, m^{*}}\right|=\min _{0 \leq k, m \leq n}\left|z-z_{k, m}\right| .
$$

Then, we observe that

$$
\left|z-z_{k^{*}, m^{*}}\right|^{\lambda}=O\left(n^{-\lambda}\right)
$$


Using this and also considering the fact that

$$
\left(\sum_{k, m=0}^{n}\left|z-z_{k, m}\right|^{-\lambda}\right)^{-1} \leq\left|z-z_{k^{*}, m^{*}}\right|^{\lambda},
$$

the proof is completed.

Now for each fixed $z \in K$ define the function $\varphi_{z}$ on $K$ by $\varphi_{z}(w):=$ $|w-z|$. Then, we also need the next lemma.

Lemma 2. For every $\lambda \geq 3$, we have

$$
\mathbb{S}_{n, \lambda}\left(\varphi_{z} ; z\right) \rightrightarrows 0 \text { on } K \text {. }
$$

Proof. Using the indices $k^{*}$ and $m^{*}$ given by (2) we may write from Lemma 1 that

$$
\begin{aligned}
\left|\mathbb{S}_{n, \lambda}\left(\varphi_{z} ; z\right)\right| \leq & \frac{\sum_{k, m=0}^{n}\left|z-z_{k, m}\right|^{1-\lambda}}{\sum_{k, m=0}^{n}\left|z-z_{k, m}\right|^{-\lambda}} \\
\leq & \left|z-z_{k^{*}, m^{*}}\right|+O\left(n^{-\lambda}\right) \sum_{k=0\left(k \neq k^{*}\right)}^{n}\left|z-z_{k, m^{*}}\right|^{1-\lambda} \\
& +O\left(n^{-\lambda}\right) \sum_{m=0\left(m \neq m^{*}\right)}^{n}\left|z-z_{k^{*}, m}\right|^{1-\lambda} \\
& +O\left(n^{-\lambda}\right) \sum_{\substack{k, m=0 \\
\left(k \neq k^{*}, m \neq m^{*}\right)}}^{n}\left|z-z_{k, m}\right|^{1-\lambda} .
\end{aligned}
$$

Hence we get

$$
\begin{aligned}
\left|\mathbb{S}_{n, \lambda}\left(\varphi_{z} ; z\right)\right| \leq & O\left(\frac{1}{n}\right)+O\left(n^{-\lambda}\right) \sum_{k=0\left(k \neq k^{*}\right)}^{n}\left|x-\frac{k}{n}\right|^{1-\lambda} \\
& +O\left(n^{-\lambda}\right) \sum_{\substack{m=0\left(m \neq m^{*}\right) \\
n}}^{n}\left|y-\frac{m}{n}\right|^{1-\lambda} \\
& +O\left(n^{-\lambda}\right) \sum_{\substack{\left.k, m=0 \\
k \neq k^{*}, m \neq m^{*}\right)}}^{n}\left|z-z_{k, m}\right|^{1-\lambda} .
\end{aligned}
$$

Since $\lambda \geq 3$, we have

$$
\begin{aligned}
\sum_{k=0\left(k \neq k^{*}\right)}^{n}\left|x-\frac{k}{n}\right|^{1-\lambda} & \leq \sum_{k=0\left(k \neq k^{*}\right)}^{n}\left(\frac{\left|k-k^{*}\right|}{n}-\frac{1}{2 n}\right)^{1-\lambda} \\
& =O\left(\frac{1}{n^{1-\lambda}}\right) \sum_{k=1}^{n} \frac{1}{k^{\lambda-1}}=O\left(\frac{1}{n^{1-\lambda}}\right) .
\end{aligned}
$$


Similarly,

$$
\sum_{m=0\left(m \neq m^{*}\right)}^{n}\left|y-\frac{m}{n}\right|^{1-\lambda}=O\left(\frac{1}{n^{1-\lambda}}\right) .
$$

Also, for $\lambda \geq 3$,

$$
\begin{aligned}
& \sum_{\substack{k, m=0 \\
\left(k \neq k^{*}, m \neq m^{*}\right)}}^{n}\left|z-z_{k, m}\right|^{1-\lambda}=\sum_{\substack{\left.k, m=0 \\
k \neq k^{*}, m \neq m^{*}\right)}}^{n}\left(\left(x-\frac{k}{n}\right)^{2}+\left(y-\frac{m}{n}\right)^{2}\right)^{(1-\lambda) / 2} \\
\leq & \sum_{\substack{\left.k, m=0 \\
k \neq k^{*}, m \neq m^{*}\right)}}^{n}\left(\left(\frac{\left|k-k^{*}\right|}{n}-\frac{1}{2 n}\right)^{2}+\left(\frac{\left|m-m^{*}\right|}{n}-\frac{1}{2 n}\right)^{2}\right)^{(1-\lambda) / 2},
\end{aligned}
$$

which implies

$$
\begin{aligned}
& \sum_{\substack{\left.k, m=0 \\
k \neq k^{*}, m \neq m^{*}\right)}}^{n}\left|z-z_{k, m}\right|^{1-\lambda} \leq \sum_{k, m=1}^{n}\left(\left(\frac{k}{n}\right)^{2}+\left(\frac{m}{n}\right)^{2}\right)^{(1-\lambda) / 2} \\
= & \frac{1}{n^{1-\lambda}}\left(\frac{1}{2^{(1-\lambda) / 2}}+2 \sum_{k=2}^{n} \frac{1}{\left(k^{2}+1\right)^{(\lambda-1) / 2}}+\sum_{k, m=2}^{n} \frac{1}{\left(k^{2}+m^{2}\right)^{(\lambda-1) / 2}}\right) .
\end{aligned}
$$

Now if $\lambda>3$, then we see

$$
\begin{aligned}
\sum_{\substack{k, m=0 \\
\left(k \neq k^{*}, m \neq m^{*}\right)}}^{n}\left|z-z_{k, m}\right|^{1-\lambda} & \leq O\left(\frac{1}{n^{1-\lambda}}\right)\left(1+\int_{1}^{n} \int_{1}^{n} \frac{d x d y}{\left(x^{2}+y^{2}\right)^{(\lambda-1) / 2}}\right) \\
& \leq O\left(\frac{1}{n^{1-\lambda}}\right)\left(1+\int_{0}^{2 \pi} \int_{1}^{\sqrt{2} n} \frac{d r d \theta}{r^{\lambda-2}}\right) \\
& =O\left(\frac{1}{n^{1-\lambda}}\right) .
\end{aligned}
$$

Then, combining the above results, for $\lambda>3$, we obtain

$$
\left|\mathbb{S}_{n, \lambda}\left(\varphi_{z} ; z\right)\right|=O\left(\frac{1}{n}\right) .
$$

Also, if $\lambda=3$, then we have

$$
\sum_{\substack{k, m=0 \\\left(k \neq k^{*}, m \neq m^{*}\right)}}^{n}\left|z-z_{k, m}\right|^{-2}=O\left(\frac{\log n}{n^{-2}}\right),
$$


which gives

$$
\left|\mathbb{S}_{n, 3}\left(\varphi_{z} ; z\right)\right|=O\left(\frac{\log n}{n}\right) .
$$

Therefore, the proof follows from (3) and (4), immediately.

Now we recall some terminology in the recent paper by Anastassiou [3].

Let $K \subset \mathbb{C}$ be a compact convex set and $L: C(K, \mathbb{C}) \rightarrow C(K, \mathbb{C})$ be a linear operator. Then, consider a positive linear operator $\tilde{L}: C(K, \mathbb{R}) \rightarrow$ $C(K, \mathbb{R})$. Assume that, for every $f \in C(K, \mathbb{C})$,

$$
|L(f ; z)| \leq \tilde{L}(|f| ; z), \quad \forall z \in K .
$$

In this case, $\tilde{L}$ is called the companion operator of $L$. Then, using the test function $e_{0}(w)=1$ and $\varphi_{z}(w)=|w-z|$, Anastassiou proved the next approximation result.

Theorem 2. (see Corollary 19 in [3]) Let $\left(L_{n}\right)$ be a sequence of linear operators from $C(K, \mathbb{C})$ into itself and $\left(\tilde{L}_{n}\right)$ be a sequence of their companion operators from $C(K, \mathbb{R})$ into itself. Additionally, assume that, for every $g \in C(K, \mathbb{R})$,

$$
L(\alpha g ; z)=\alpha \tilde{L}(g ; z), \forall z \in K, \forall \alpha \in \mathbb{C} .
$$

Then, for every $f \in C(K, \mathbb{C})$, we have

$$
\left\|L_{n}(f)-f\right\| \leq\|f\|\left\|\tilde{L}_{n}\left(e_{0}\right)-e_{0}\right\|+\left(1+\left\|\tilde{L}_{n}\left(e_{0}\right)\right\|\right) \omega\left(f,\left\|\tilde{L}_{n}\left(\varphi_{z}\right)\right\|\right),
$$

where $\|\cdot\|$ denotes the usual supremum norm on $K$ and $\omega(f, \delta) \quad(\delta>0)$ is the modulus of continuity of $f$ on $K$. In this case, if

$$
\tilde{L}_{n}\left(e_{0}\right) \rightrightarrows e_{0} \text { and } \tilde{L}_{n}\left(\varphi_{z} ; z\right) \rightrightarrows 0 \text { on } K,
$$

then, for every $f \in C(K, \mathbb{C})$, we have

$$
L_{n}(f) \rightrightarrows f \text { on } K .
$$

Now we are ready to prove Theorem 1.

Proof of Theorem 1. Now taking $K=[0,1] \times[0,1]$ and $\lambda>0$, consider following operators on $C(K, \mathbb{R})$

$$
\tilde{\mathbb{S}}_{n, \lambda}(f ; z)=\frac{\sum_{k, m=0}^{n}\left|z-z_{k, m}\right|^{-\lambda} f\left(z_{k, m}\right)}{\sum_{k, m=0}^{n}\left|z-z_{k, m}\right|^{-\lambda}} .
$$

Then, it is easy to check from (5) that, for each $n \in \mathbb{N}$ and $\lambda>0, \tilde{\mathbb{S}}_{n, \lambda}$ is the companion operator of $\mathbb{S}_{n, \lambda}$. Also, these operators satisfy the assumption (6). We observe that $\tilde{\mathbb{S}}_{n, \lambda}\left(\varphi_{z}\right)=\mathbb{S}_{n, \lambda}\left(\varphi_{z}\right)$ since $\varphi_{z}$ is a real-valued function. Therefore, using the fact that $\widetilde{\mathbb{S}}_{n, \lambda}\left(e_{0}\right)=e_{0}=1$ on $K$ and taking into account Lemma 2 , all conditions of Theorem 2 hold for $\lambda \geq 3$, which immediately gives the proof of Theorem 1 . 
Now we give two applications on the set $K=[0,1] \times[0,1]$.

Example 1. Define the function $f$ by

$$
f(z)=i \exp \left(2\left(\bar{z}^{2}-z^{2}\right)\right), \quad z \in K,
$$

where $\bar{z}$ denotes the conjugate of $z$ and $\exp (\cdot)$ is the complex exponential function. Since $f \in C(K, \mathbb{C})$, it follows from Theorem 1 that, for every $\lambda \geq 3$,

$$
\mathbb{S}_{n, \lambda}(f) \rightrightarrows f \text { on } K \text {. }
$$

To visualize this uniform approximation we may consider the real or imaginary part of the function $f$. Then, for example, it is easy to see that

$$
\Re\left(\mathbb{S}_{n, \lambda}(f)\right) \rightrightarrows \Re(f) \text { on } K,
$$

which is indicated in Fig. 2 for the values $\lambda=3.7$ and $n=8,15,30$.

Example 2. Define the function $g$ by

$$
g(z)=z^{3}-z^{2}+1-\frac{i}{5}, \quad z \in K .
$$

Then, Theorem 1 implies that, for every $\lambda \geq 3$,

$$
\mathbb{S}_{n, \lambda}(g) \rightrightarrows g \text { on } K
$$

holds, which also gives

$$
\left|\mathbb{S}_{n, \lambda}(g)\right| \rightrightarrows|g| \text { on } K \text {. }
$$

This approximation is indicated in Fig. 3 via the contour lines, where larger values are colored lighter. In Fig. 3 we use the parameter values $\lambda=5$ and $n=10,20,50$.

\section{Summability by the Complex Shepard Operators}

Now let $\left(u_{n}\right)$ be a sequence of complex functions from $K$ into itself. Then we consider the following slight modification of the complex Shepard operators:

$$
\mathbb{S}_{n, \lambda}^{*}(f ; z)=\mathbb{S}_{n, \lambda}\left(f ; u_{n}(z)\right)
$$

for $z \in K, n \in \mathbb{N}, \lambda>0$ and $f \in C(K, \mathbb{C})$. Defining the function $e_{1}(z)=z$ on $K$, we get the next approximation theorem.

Theorem 3. If $u_{n} \rightrightarrows e_{1}$ on $K$, then, for every $f \in C(K, \mathbb{C})$ and $\lambda \geq 3$, we have $\mathbb{S}_{n, \lambda}^{*}(f) \rightrightarrows f$ on $K$.

Proof. From the definitions of (1) and (10), we may write that

$$
\left|\mathbb{S}_{n, \lambda}^{*}(f ; z)-f(z)\right| \leq\left|\mathbb{S}_{n, \lambda}\left(f ; u_{n}(z)\right)-f\left(u_{n}(z)\right)\right|+\left|f\left(u_{n}(z)\right)-f(z)\right|
$$

for any $f \in C(K, \mathbb{C})$. Then, using (3), (4) and (7) we get

$$
\left|\mathbb{S}_{n, \lambda}^{*}(f ; z)-f(z)\right|=O\left(\omega\left(f, \frac{1}{n}\right)\right)+\left|f\left(u_{n}(z)\right)-f(z)\right| \quad \text { for } \lambda>3
$$




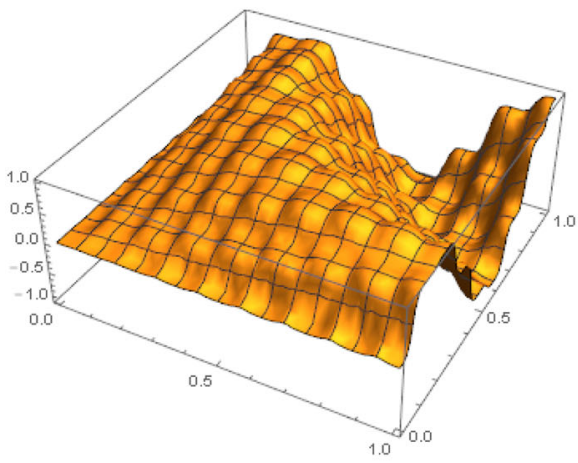

(a) $n=8$

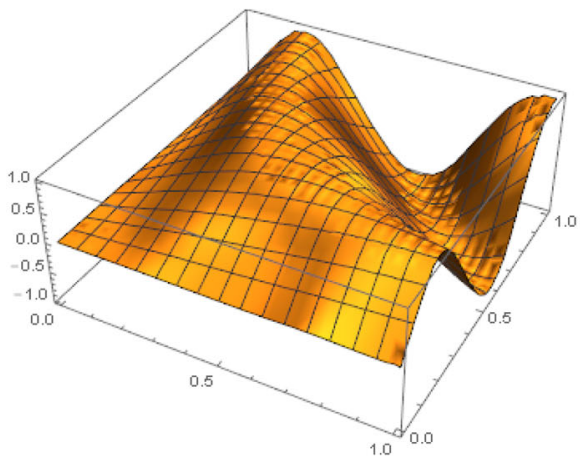

(c) $n=30$

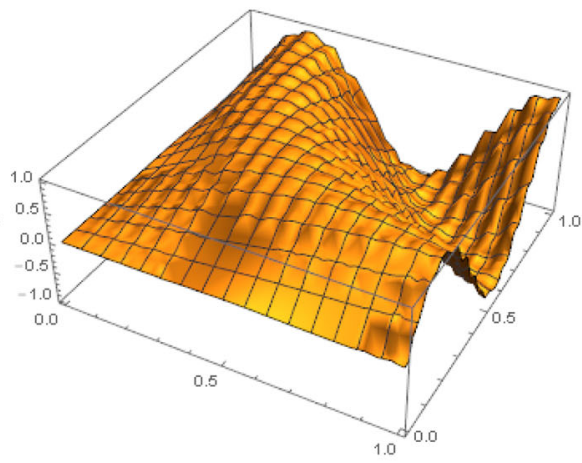

(b) $n=15$

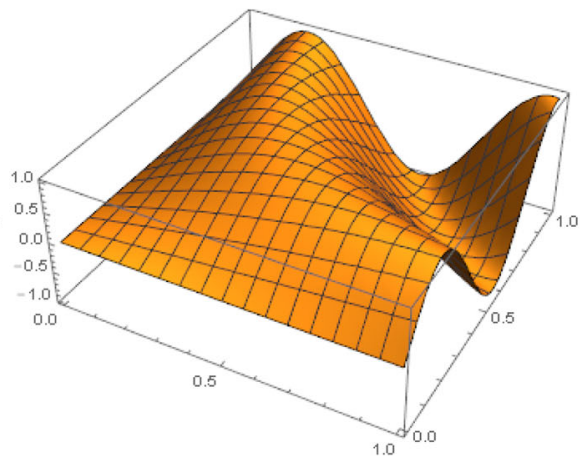

(d) $\Re(f)$

Figure 2. Approximation to the real part of the function $f$ given by (8) by means of the real part of the operators $\mathbb{S}_{n, \lambda}(f)$ for the values $\lambda=3.7$ and $n=8,15,30$

and

$$
\left|\mathbb{S}_{n, \lambda}^{*}(f ; z)-f(z)\right|=O\left(\omega\left(f, \frac{\log n}{n}\right)\right)+\left|f\left(u_{n}(z)\right)-f(z)\right|
$$

for $\lambda=3$. Since $u_{n} \rightrightarrows e_{1}$ on $K$ and $f$ is uniformly continuous on $K$, we immediately observe that, for every $\lambda \geq 3$,

$$
\mathbb{S}_{n, \lambda}^{*}(f) \rightrightarrows f \text { on } K,
$$

which completes the proof.

Then, it is natural to arise the following problem.

Problem 1. What should we do in order to preserve the approximation in Theorem 3 in "some sense" if the ordinary convergence of the function sequence $\left(u_{n}\right)$ to the function $e_{1}$ fails? 


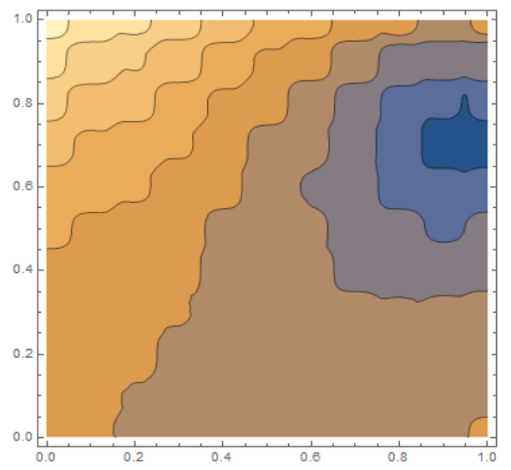

(a) $n=10$

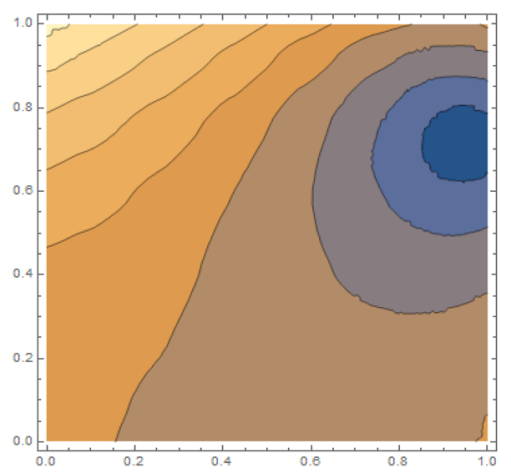

(c) $n=50$

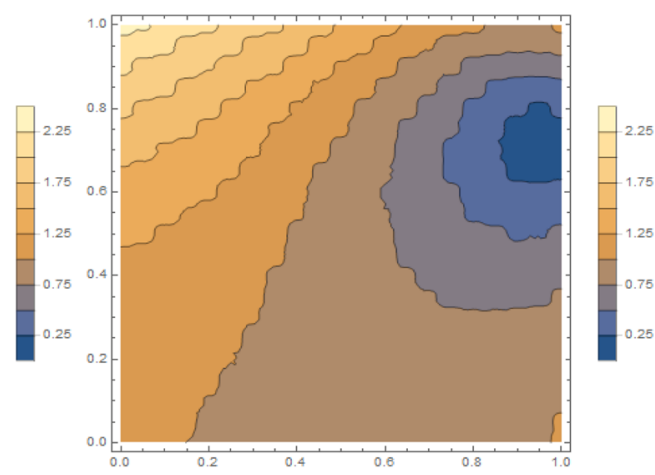

(b) $n=20$

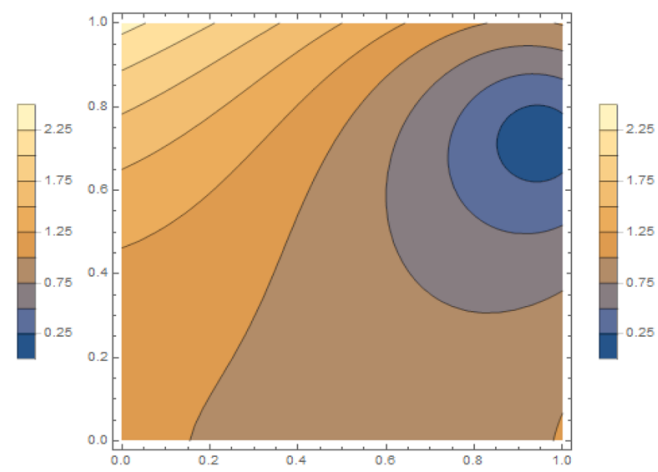

(d) $|g|$

Figure 3. Contour lines of $\left|\mathbb{S}_{n, \lambda}(g)\right|$ and $|g|$ for the values $\lambda=5$ and $n=10,20,50$, where $g$ is given by (9)

To find an affirmative answer to Problem 1, we will mainly consider nonnegative regular summability matrices. It is known that a summability method is a common and useful way to overcome the lack of the usual convergence $[8,26]$. Recent studies demonstrate that it is also quite effective in the classical approximation theory (see, for instance, $[1,4,5,23,25,28,30,32])$. We now use it in the approximation by complex Shepard operators.

Recall that, for a given infinite matrix $A:=\left[a_{j n}\right](j, n \in \mathbb{N})$ and a sequence $x:=\left(x_{n}\right)$, the $A$-transformed sequence of $\left(x_{n}\right)$ is given by $A x:=$ $\left((A x)_{j}\right)=\sum_{n=1}^{\infty} a_{j n} x_{n}$ provided that the series is convergent for every $j \in$ $\mathbb{N}$. In this case, we say that $A$ is a summability matrix method. If the $A$ transformed sequence of $\left(x_{n}\right)$ converges to a number $L$, then the sequence $\left(x_{n}\right)$ is said to be $A$-summable (or $A$-convergent) to $L$, which is denoted by $x_{n} \stackrel{A}{\rightarrow} L$ or $A$-lim $x=L$. It is also possible to give the same definition for 
a sequence of functions. Let $\left(f_{n}\right)$ be a sequence of complex-valued functions defined on a set $D \subset \mathbb{C}$. Then, we say that $\left(f_{n}\right)$ is uniformly $A$-summable to a function $f$ on $D$ if

$$
\lim _{j \rightarrow \infty} \sum_{n=1}^{\infty} a_{j n} f_{n}(z)=f(z), \text { uniformly in } z \in D,
$$

which is denoted by $f_{n} \stackrel{A}{\rightrightarrows} f$ on $D$. A regular matrix summability method is a matrix transformation of a convergent sequence which preserves the limit. It is well-known that the Silverman-Toeplitz theorem characterizes the regularity of a matrix summability method (see $[8,26]$ ).

Throughout the paper, we will consider $A=\left[a_{j n}\right]$ as a nonnegative regular summability matrix. By the nonnegativity of a matrix method, we mean all entries of the matrix are nonnegative.

Then, the first idea that comes to mind regarding the solution of Problem 1 is as follows.

$$
\text { Claim: If } u_{n} \stackrel{A}{\rightrightarrows} e_{1} \text { on } K, \text { then } \mathbb{S}_{n, \lambda}^{*}(f) \stackrel{A}{\rightrightarrows} f \text { on } K
$$

holds for every $f \in C(K, \mathbb{C})$ and $\lambda \geq 3$. However, the next example shows that the claim in (13) is not satisfied for the function $e_{2}(z)=z^{2}$ on $K$.

Example 3. Define the function sequence $\left(u_{n}\right)$ on the set $K$ by

$$
u_{n}(z)=\left\{\begin{array}{c}
2 z, \text { if } n \text { is odd } \\
0, \text { if } n \text { is even. }
\end{array}\right.
$$

Assume that $A=\left[a_{j n}\right]$ is any nonnegative regular summability matrix such that

$$
u_{n} \stackrel{A}{\rightrightarrows} e_{1} \text { on } K \text {. }
$$

It follows from (14) that

$$
\left|\sum_{n=1}^{\infty} a_{j n} u_{n}(z)-e_{1}(z)\right|=2|z|\left|\sum_{n=1}^{\infty} a_{j, 2 n-1}-\frac{1}{2}\right| .
$$

Taking supremum over $z \in K$, we get

$$
\left\|\sum_{n=1}^{\infty} a_{j n} u_{n}-e_{1}\right\|=2 \sqrt{2}\left|\sum_{n=1}^{\infty} a_{j, 2 n-1}-\frac{1}{2}\right| .
$$

Since $u_{n} \stackrel{A}{\rightrightarrows} e_{1}$ on $K$, we see that

$$
\lim _{j \rightarrow \infty} \sum_{n=1}^{\infty} a_{j, 2 n-1}=\frac{1}{2}
$$


On the other hand, we may write from (14) that

$$
e_{2}\left(u_{n}(z)\right)=\left\{\begin{array}{c}
4 z^{2}, \text { if } n \text { is odd } \\
0, \text { if } n \text { is even. }
\end{array}\right.
$$

Then, defining the function $h(z)=2 z^{2}$ on $K$, we see that

$$
\begin{aligned}
\left|\sum_{n=1}^{\infty} a_{j n} \mathbb{S}_{n, \lambda}^{*}\left(e_{2} ; z\right)-h(z)\right| \leq & \left|\sum_{n=1}^{\infty} a_{j n} \mathbb{S}_{n, \lambda}\left(e_{2} ; u_{n}(z)\right)-\sum_{n=1}^{\infty} a_{j n} e_{2}\left(u_{n}(z)\right)\right| \\
& +\left|\sum_{n=1}^{\infty} a_{j n} e_{2}\left(u_{n}(z)\right)-h(z)\right| \\
\leq & \sum_{n=1}^{\infty} a_{j n}\left|\mathbb{S}_{n, \lambda}\left(e_{2} ; u_{n}(z)\right)-e_{2}\left(u_{n}(z)\right)\right| \\
& +4|z|^{2}\left|\sum_{n=1}^{\infty} a_{j, 2 n-1}-\frac{1}{2}\right|
\end{aligned}
$$

Therefore, we get from (3), (4) and (7) that

$$
\left\|\sum_{n=1}^{\infty} a_{j n} \mathbb{S}_{n, \lambda}^{*}\left(e_{2}\right)-h\right\|=O\left(\sum_{n=1}^{\infty} a_{j n} \omega\left(f, \frac{1}{n}\right)\right)+8\left|\sum_{n=1}^{\infty} a_{j, 2 n-1}-\frac{1}{2}\right|
$$

for $\lambda>3$; and

$$
\left\|\sum_{n=1}^{\infty} a_{j n} \mathbb{S}_{n, \lambda}^{*}\left(e_{2}\right)-h\right\|=O\left(\sum_{n=1}^{\infty} a_{j n} \omega\left(f, \frac{\log n}{n}\right)\right)+8\left|\sum_{n=1}^{\infty} a_{j, 2 n-1}-\frac{1}{2}\right|
$$

for $\lambda=3$. Using (15) in the last two inequalities and also considering the regularity of $A$, we obtain

$$
\mathbb{S}_{n, \lambda}^{*}\left(e_{2}\right) \stackrel{A}{\rightrightarrows} h \neq e_{2} \text { on } K
$$

for every $\lambda \geq 3$. Hence, (13) does not work for the test function $e_{2}$.

We understand from Example 3 that in order to find a solution to Problem 1, we need a slightly stronger concept than the $A$-summability. In fact, there is such a concept in the summability theory, the so-called "strong summability".

Recall that, for a given summability matrix $A=\left[a_{j n}\right]$, a sequence $x=$ $\left(x_{n}\right)$ is said to be strongly $A$-summable to a value $L$ if all sums $\sum_{n=1}^{\infty} a_{j n}\left|x_{n}-L\right|$ $(j=1,2, \ldots)$ exist and converges to zero as $j \rightarrow \infty$. We denote this by $x_{n} \stackrel{|A|}{\rightarrow} L$. The concept of strong summability can be also given for a sequence of functions. Let $\left(f_{n}\right)$ be a sequence of complex-valued functions defined on a set $D \subset \mathbb{C}$. Then, we say that $\left(f_{n}\right)$ is strongly uniform $A$-summable to a function $f$ on $D$ provided that 


$$
\lim _{j \rightarrow \infty} \sum_{n=1}^{\infty} a_{j n}\left|f_{n}(z)-f(z)\right|=0, \text { uniformly in } z \in D,
$$

which is denoted by

$$
f_{n} \stackrel{|A|}{\rightrightarrows} f \text { on } D .
$$

Since strong $A$-summability implies $A$-summability for any nonnegative regular matrix $A$, we may write, for bounded functions on $D$, that

$$
f_{n} \rightrightarrows f \text { on } D \Rightarrow f_{n} \stackrel{|A|}{\rightrightarrows} f \text { on } D \Rightarrow f_{n} \stackrel{A}{\rightrightarrows} f \text { on } D .
$$

But the converse is not always true. For example, observe that the function sequence $\left(u_{n}\right)$ in Example 3 is (uniform) $C_{1}$-summable to $e_{1}$ but not strongly.

Theorem 4. Let $A=\left[a_{j n}\right]$ be a nonnegative regular matrix method and $\left(u_{n}\right)$ be a sequence of complex-valued functions defined on $K$ such that

$$
u_{n} \stackrel{|A|}{\rightrightarrows} e_{1} \text { on } K .
$$

Then, for every $f \in C(K, \mathbb{C})$ and $\lambda \geq 3$, we have

$$
\mathbb{S}_{n, \lambda}^{*}(f) \stackrel{|A|}{\rightrightarrows} f \text { on } K .
$$

Proof. Let $f \in C(K, \mathbb{C})$ be given. Then, from the uniform continuity of $f$ on the convex set $K$, the function $f$ satisfies the almost Lipschitz property on $K$ (see [33]), that is, for every $\varepsilon>0$, there exists a positive constant $M_{\varepsilon}$ such that

$$
|f(s)-f(z)| \leq M_{\varepsilon}|s-z|+\varepsilon
$$

holds for all $s, z \in K$. We may write from (11) and (18) that, for $\lambda>3$

$$
\begin{aligned}
\sum_{n=1}^{\infty} a_{j n}\left|\mathbb{S}_{n, \lambda}^{*}(f ; z)-f(z)\right|= & O\left(\sum_{n=1}^{\infty} a_{j n} \omega\left(f, \frac{1}{n}\right)\right) \\
& +M_{\varepsilon} \sum_{n=1}^{\infty} a_{j n}\left(\left|u_{n}(z)-z\right|+\varepsilon\right) .
\end{aligned}
$$

From the hypothesis (16) and the regularity of the method $A$, the right-hand side of the last inequality goes to zero uniformly as $j \rightarrow \infty$, which means

$$
\mathbb{S}_{n, \lambda}^{*}(f) \stackrel{|A|}{\rightrightarrows} f \text { on } K \text { for every } \lambda>3 .
$$

Similarly, one can easily obtain from (12) and (18) that

$$
\mathbb{S}_{n, \lambda}^{*}(f) \stackrel{|A|}{\rightrightarrows} f \text { on } K \text { for } \lambda=3 .
$$

Hence, the proof is completed. 
We should note that, under the same condition in Theorem 4 , the $A$ strong summability in (17) also implies

$$
\mathbb{S}_{n, \lambda}^{*}(f) \stackrel{A}{\rightrightarrows} f \text { on } K \text { for every } \lambda \geq 3 .
$$

Example 4. Define the function sequence $\left(u_{n}\right)$ on $K$ by

$$
u_{n}(z)= \begin{cases}z+1, & \text { if } n=m^{2}(m \in \mathbb{N}) \\ z, & \text { otherwise. }\end{cases}
$$

Now consider the Cesàro method $C_{1}=\left[c_{j n}\right]$ given by

$$
c_{j n}=\left\{\begin{array}{l}
\frac{1}{j}, \text { if } n=1,2, \ldots, j \\
0, \text { otherwise. }
\end{array}\right.
$$

Then, we get from (19) that

$$
\begin{aligned}
\sum_{n=1}^{\infty} c_{j n}\left|u_{n}(z)-e_{1}(z)\right| & =\frac{1}{j} \sum_{n=1}^{j}\left|u_{n}(z)-z\right| \\
& \leq \frac{1}{\sqrt{j}}
\end{aligned}
$$

which implies

$$
u_{n} \stackrel{\left|C_{1}\right|}{\rightrightarrows} e_{1} \text { on } K
$$

Therefore, by Theorem 1, for the corresponding (modified) complex Shepard operators, we have, for every $f \in C(K, \mathbb{C})$ and $\lambda \geq 3$,

$$
\mathbb{S}_{n, \lambda}^{*}(f) \stackrel{\left|C_{1}\right|}{\rightrightarrows} f \text { on } K
$$

which also gives

$$
\mathbb{S}_{n, \lambda}^{*}(f) \stackrel{C_{1}}{\rightrightarrows} f \text { on } K .
$$

However, observe that it is not possible to approximate $f$ (in the ordinary sense) by means of $\mathbb{S}_{n, \lambda}^{*}(f)$ for any function $f$ satisfying $f(z+1) \neq f(z)$ for some $z \in K$. We also understand from (20) that the arithmetic mean of (modified) complex Shepard operators is uniformly convergent to $f$ on the set $K$, that is, for every $\lambda \geq 3$

$$
\frac{\mathbb{S}_{1, \lambda}\left(f ; u_{1}(z)\right)+\mathbb{S}_{2, \lambda}\left(f ; u_{2}(z)\right)+\cdots+\mathbb{S}_{j, \lambda}\left(f ; u_{j}(z)\right)}{j} \rightrightarrows f(z) \text { on } K .
$$

Hence, one can easily check that

$$
\frac{\Im\left\{\mathbb{S}_{1, \lambda}\left(f ; u_{1}(z)\right)\right\}+\Im\left\{\mathbb{S}_{2, \lambda}\left(f ; u_{2}(z)\right)\right\}+\cdots+\Im\left\{\mathbb{S}_{j, \lambda}\left(f ; u_{j}(z)\right)\right\}}{j} \rightrightarrows \Im\{f(z)\},
$$




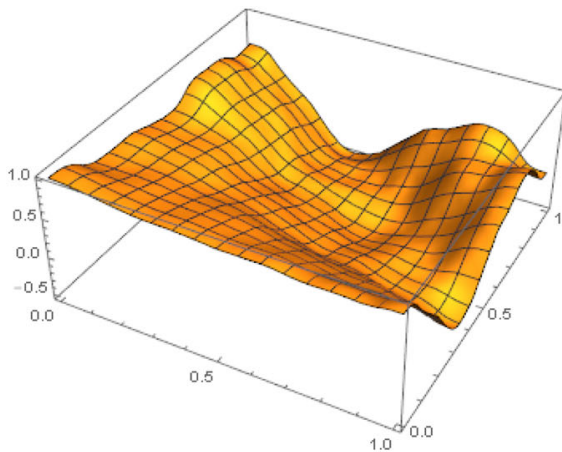

(a) $\lambda=3$ and $j=6$

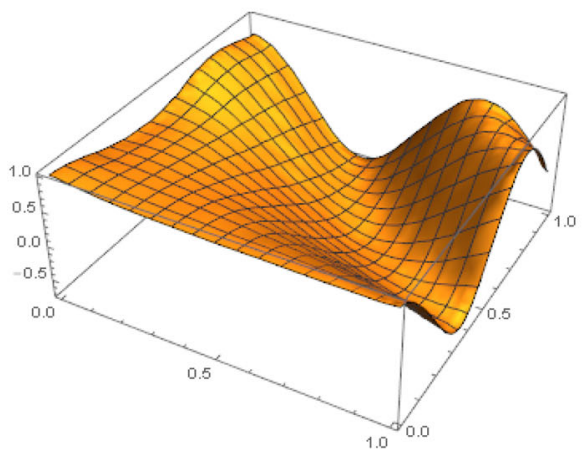

(c) $\lambda=3$ and $j=25$

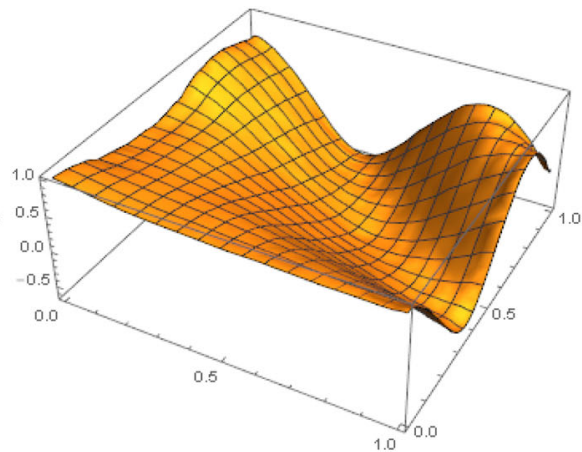

(b) $\lambda=3$ and $j=16$

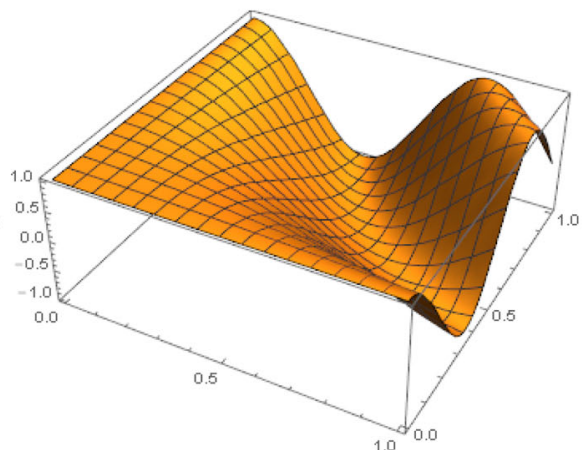

(d) $\mathfrak{I}(f)$

Figure 4. Cesàro approximation to the imaginary part of the function $f$ given by (8) by means of the imaginary part of the modified complex Shepard operators

uniformly with respect to $z \in K$. This (uniform) Cesàro approximation is indicated in Fig. 4 for the function $f$ given by (8) and the parameter values $\lambda=3, j=6,16,25$.

\section{Concluding Remarks}

In this study, we have introduced the complex Shepard operators $\mathbb{S}_{n, \lambda}$ in order to approximate continuous and complex-valued function on the unit square $K=[0,1] \times[0,1]$. We have also examined the effects of regular summability methods on the approximation by these operators. On the unit square, we have considered the sample points $z_{k, m}=\frac{k}{n}+i \frac{m}{n}(k, m=0,1, \ldots, n)$. We should note that the region $K$ can be extended to any compact rectangular region 


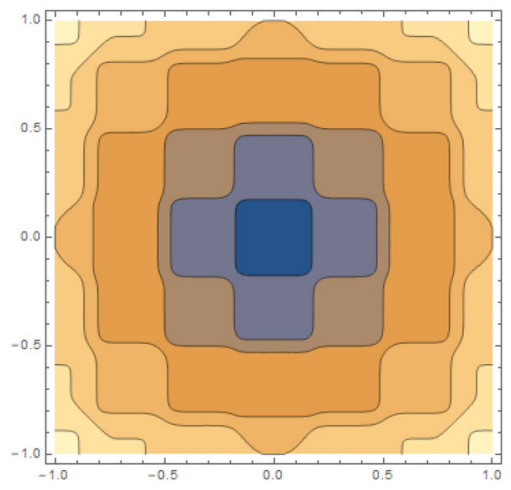

(a) $n=6$

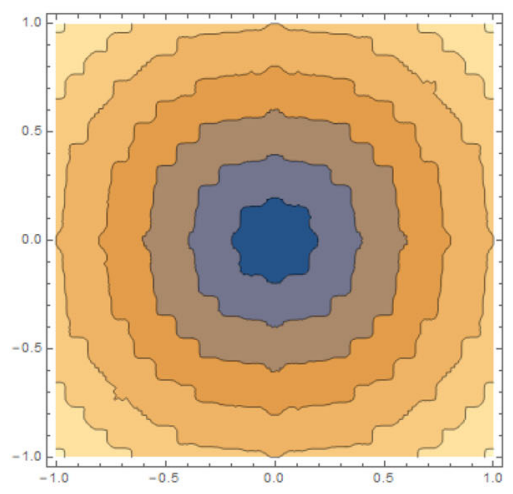

(c) $n=20$

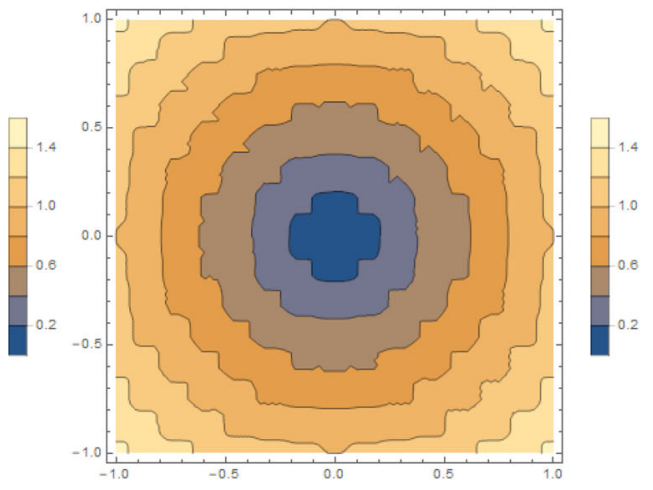

(b) $n=14$

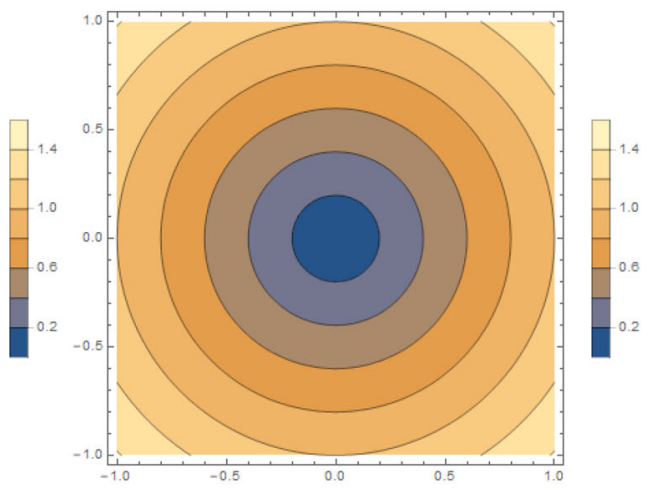

(d) $\left|e_{1}\right|$

Figure 5. Contour lines of $\left|\mathbb{S}_{n, \lambda}^{R}\left(e_{1}\right)\right|$ and $\left|e_{1}\right|$ for the values $\lambda=4$ and $n=6,14,20$, where $R=[-1,1] \times[-1,1]$

$R=[a, b] \times[c, d]$ with $a<b$ and $c<d$. Indeed, for a given continuous function $f: R \rightarrow \mathbb{C}$, choosing the sample points

$$
z_{k, m}^{R}:=\left(a+\frac{(b-a) k}{n}\right)+i\left(c+\frac{(d-c) m}{n}\right), \quad k, m=0,1, \ldots, n,
$$

we can modify the complex Shepard operators as follows:

$$
\mathbb{S}_{n, \lambda}^{R}(f ; z)=\frac{\sum_{k, m=0}^{n}\left|z-z_{k, m}^{R}\right|^{-\lambda} f\left(z_{k, m}^{R}\right)}{\sum_{k, m=0}^{n}\left|z-z_{k, m}^{R}\right|^{-\lambda}}, \quad \lambda>0, n \in \mathbb{N}, z \in R .
$$


In this case, all the results obtained in the previous sections are also valid on the region $R$. Hence, from Theorem 1, we may write that

$$
\mathbb{S}_{n, \lambda}^{R}(f) \rightrightarrows f
$$

holds for every $f \in C(R, \mathbb{C})$ and $\lambda \geq 3$. The approximation in (22) also implies

$$
\left|\mathbb{S}_{n, \lambda}^{R}(f)\right| \rightrightarrows|f|,
$$

which is indicated in Fig. 5 for the function $e_{1}(z)=z$ by taking $R=[-1,1] \times$ $[-1,1]$ and also using the contour lines.

Finally, for a future work, it would be interesting to modify the complex Shepard operators in order to approximate functions that do not need to be continuous (for example, integrable functions). These types of operators are known in the literature as Kantorovich operators and have some applications in image/signal processing and sampling theory (see [11-13]).

\section{Acknowledgements}

The authors are grateful to the referees for their interesting remarks.

Funding Open access funding provided by Universitá degli Studi di Roma La Sapienza within the CRUI-CARE Agreement.

\section{Declarations}

Conflict of interest The authors declare that they have no conflict of interest.

Open Access. This article is licensed under a Creative Commons Attribution 4.0 International License, which permits use, sharing, adaptation, distribution and reproduction in any medium or format, as long as you give appropriate credit to the original author(s) and the source, provide a link to the Creative Commons licence, and indicate if changes were made. The images or other third party material in this article are included in the article's Creative Commons licence, unless indicated otherwise in a credit line to the material. If material is not included in the article's Creative Commons licence and your intended use is not permitted by statutory regulation or exceeds the permitted use, you will need to obtain permission directly from the copyright holder. To view a copy of this licence, visit http://creativecommons. org/licenses/by/4.0/.

\section{References}

[1] Alemdar, M.E., Duman, O.: General summability methods in the approximation by Bernstein-Chlodovsky operators. Numer. Funct. Anal. Optim. 42(5), 497-509 (2021). https://doi.org/10.1080/01630563.2021.1895831

[2] Amato, U., Della Vecchia, B.: Weighting Shepard-type operators. Comput. Appl. Math. 36(2), 885-902 (2017). https://doi.org/10.1007/s40314-015-0263-y 
[3] Anastassiou, G.A.: Complex Korovkin theory. J. Comput. Anal. Appl. 28(6), 981-996 (2020)

[4] Aslan, I., Duman, O.: Approximation by nonlinear integral operators via summability process. Math. Nachr. 293(3), 430-448 (2020). https://doi.org/10.1002/ mana.201800187

[5] Atlihan, O.G., Orhan, C.: Summation process of positive linear operators. Comput. Math. Appl. 56(5), 1188-1195 (2008). https://doi.org/10.1016/j.camwa. 2008.02.020

[6] Barnhill, R., Dube, R., Little, F.: Properties of Shepards surfaces. Rocky Mt. J. Math. 13(2), 365-382 (1983). https://doi.org/10.1216/RMJ-1983-13-2-365

[7] Barnhill, R., Ou, H.: Surfaces defined on surfaces. Comput. Aided Geom. Des. 7(1), 323-336 (1990). https://doi.org/10.1016/0167-8396(90)90040-X

[8] Boos, J.: Classical and Modern Methods in Summability. Oxford University Press, Oxford (2000)

[9] Caira, R., Dell'Accio, F.: Shepard-Bernoulli operators. Math. Comput. 76(257), 299-321 (2008). https://doi.org/10.1090/S0025-5718-06-01894-1

[10] Cavoretto, R., De Rossi, A., Dell'Accio, F., Di Tommaso, F.: An efficient trivariate algorithm for tetrahedral Shepard interpolation. J. Sci. Comput. 82(3), 57 (2020). https://doi.org/10.1007/s10915-020-01159-3

[11] Coroianu, L., Costarelli, D., Gal, S.G., Vinti, G.: Approximation by multivariate max-product Kantorovich-type operators and learning rates of least-squares regularized regression. Commun. Pure Appl. Anal. 19(8), 4213-4225 (2020). https://doi.org/10.3934/cpaa.2020189

[12] Costarelli, D., Vinti, G.: Approximation by max-product neural network operators of Kantorovich type. Results Math. 69(3), 505-519 (2016). https://doi.org/ 10.1007/s00025-016-0546-7

[13] Costarelli, D., Vinti, G.: An inverse result of approximation by sampling Kantorovich series. Proc. Edinb. Math. Soc. 62(1), 265-280 (2019). https://doi.org/ 10.1017/S0013091518000342

[14] Criscuolo, G., Mastroianni, G.: Estimates of the Shepard interpolatory procedure. Acta Math. Hung. 61(1-2), 79-91 (1993). https://doi.org/10.1007/ bf01872100

[15] Della Vecchia, B.: Direct and converse results by rational operators. Constr. Approx. 12(2), 271 (1996). https://doi.org/10.1007/BF02433043

[16] Della Vecchia, B., Mastroianni, G.: Pointwise simultaneous approximation by rational operators. J. Approx. Theory 65(2), 140-150 (1991). https://doi.org/ 10.1016/0021-9045(91)90099-V

[17] Dell'Accio, F., Di Tommaso, F.: Complete Hermite Birkhoff interpolation on scattered data by combined Shepard operators. J. Comput. Appl. Math. 300, 192-206 (2016). https://doi.org/10.1016/j.cam.2015.12.016

[18] Dell'Accio, F., Di Tommaso, F.: Scattered data interpolation by Shepards like methods: classical results and recent advances. Dolomites Res. Notes Approx. 9, $32-44(2016)$ 
[19] Dell'Accio, F., Di Tommasco, F.: Rate of convergence of multinode Shepard operators. Dolomites Res. Notes Approx. 12(1), 1-6 (2019). https://doi.org/10. 14658/pupj-drna-2019-1-1

[20] Dell'Accio, F., Di Tommasco, F.: On the hexagonal Shepard method. Appl. Numer. Math. 150, 51-64 (2020). https://doi.org/10.1016/j.apnum.2019.09.005

[21] Dell'Accio, F., Di Tommaso, F., Hormann, K.: On the approximation order of triangular Shepard interpolation. IMA J. Numer. Anal. 36(1), 359-379 (2016). https://doi.org/10.1093/imanum/dru065

[22] Dell'Accio, F., Di Tommaso, F., Nouisser, O., Siar, N.: Solving Poisson equation with Dirichlet conditions through multinode Shepard operators. Comput. Math. Appl. 98, 254-260 (2021). https://doi.org/10.1016/j.camwa.2021.07.021

[23] Demirci, K., Boccuto, A., Yıldız, S., Dirik, F.: Relative uniform convergence of a sequence of functions at a point and Korovkin-type approximation theorems. Positivity 24(1), 1-11 (2020). https://doi.org/10.1007/s11117-019-00656-6

[24] Farwig, R.: Rate of convergence of Shepards global interpolation formula. Math. Comput. 46(174), 577-590 (1986). https://doi.org/10.1090/ S0025-5718-1986-0829627-0

[25] Gökçer, T.Y., Duman, O.: Regular summability methods in the approximation by max-min operators. Fuzzy Sets Syst. (2021). https://doi.org/10.1016/j.fss. 2021.03.003

[26] Hardy, G.H.: Divergent Series. Clarendon Press, Oxford (1949)

[27] Hermann, T.: Rational interpolation of periodic functions. Rendiconti del Circolo Matematico di Palermo (2) Supplemento 33, 337-344 (1993)

[28] Mohapatra, R.N.: Quantitative results on almost convergence of a sequence of positive linear operators. J. Approx. Theory 20(3), 239-250 (1977). https://doi. org/10.1016/0021-9045(77)90058-2

[29] Shepard, D.: A two-dimensional interpolation function for irregularly-spaced data. In: Proceedings of the 23rd ACM National Conference, pp. 517-524. ACM, New York, NY (1968)

[30] Swetits, J.: On summability and positive linear operators. J. Approx. Theory 25(2), 186-188 (1979). https://doi.org/10.1016/0021-9045(79)90008-X

[31] Szabados, J.: Direct and converse approximation theorems for the Shepard operator. Approx. Theory Appl. 7(3), 63-76 (1991). https://doi.org/10.1007/ BF02836457

[32] Turkun, C., Duman, O.: Modified neural network operators and their convergence properties with summability methods. Rev. Real Acad. Ciencias Exactas Físicas Nat. Ser. A. Matemáticas 114(3), 132 (2020). https://doi.org/10.1007/ s13398-020-00860-0

[33] Vanderbei, R.J.: Uniform continuity is almost Lipschitz continuity. Statistics and Operations Research Series SOR-91 11. Princeton University (1991). https:// vanderbei.princeton.edu/tex/unif_cont/uc3.pdf

[34] Yu, D.: On weighted approximation by rational operators for functions with singularities. Acta Math. Hung. 136(1), 56-75 (2012). https://doi.org/10.1007/ s10474-011-0187-y 
[35] Yu, D., Zhou, S.: Approximation by rational operators in $L^{p}$ spaces. Math. Nachr. 282(11), 1600-1618 (2009). https://doi.org/10.1002/mana.200610812

[36] Zhou, X.: The saturation class of Shepard operators. Acta Math. Hung. 80(4), 293-310 (1998). https://doi.org/10.1023/A:1006538323418

\section{Oktay Duman}

Department of Mathematics

TOBB Economics and Technology University

Ankara

Turkey

e-mail: oduman@etu.edu.tr

Biancamaria Della Vecchia

Dipartimento di Matematica

Università di Roma 'La Sapienza'

Rome

Italy

e-mail: biancamaria.dellavecchia@uniroma1.it

Received: May 20, 2021.

Accepted: September 13, 2021.

Publisher's Note Springer Nature remains neutral with regard to jurisdictional claims in published maps and institutional affiliations. 